\title{
Phase Transitions in Nanomaterials using Movie Mode Dynamic Transmission Electron Microscopy
}

\author{
B. W. Reed ${ }^{1}$, T. LaGrange ${ }^{1}$, J. T McKeown ${ }^{1}$, M. K. Santala ${ }^{1}$, G. H. Campbell ${ }^{1}$, and K. J. Koski ${ }^{2}$ \\ 1. Physical and Life Sciences Directorate, Lawrence Livermore National Laboratory, Livermore, CA, \\ USA \\ 2. Department of Chemistry, Brown University, Providence, RI, USA
}

2D layered nanomaterials including graphene, $\mathrm{Bi}_{2} \mathrm{Se}_{3}, \mathrm{MoO}_{3}$, and many others, have attracted enormous interest for both their countless industrial uses and their seemingly boundless ability to produce fascinating and unexpected physical behavior. This broad class of materials exhibits extraordinary electronic properties including Dirac-cone dispersion relations and topological insulating behavior while also being ideal for such literally down-to-earth applications as solid lubricants in fossil fuel drilling. 2D layered nanomaterials have also proven capable of reversibly absorbing surprisingly large amounts of foreign material within the van der Waals gaps separating the layers - which is to say, they are nearly ideal hosts for chemical intercalation.

Recent studies [1,2] have shown that relatively simple chemical methods can be used to intercalate very high densities of zero-valent metal atoms into many of these materials. In some cases the intercalation density is so high that the intercalated atoms outnumber the original host atoms. Yet this occurs with little or no disruption of the crystal lattice and, in many cases, the charge transfer between the host and the intercalant is immeasurably small. Especially intriguing is the variety of ordered and disordered phases exhibited by the intercalated materials, as revealed by electron diffraction coupled with in situ heating [2].

In the present study, we untangle some of these phase transitions as exhibited by $\mathrm{MoO}_{3}$ nanoribbons intercalated with roughly $10 \%$ atomic copper content. Specifically, we show that the as-fabricated structure is unstable and can be thermally annealed into two completely different structures depending on the time scale of the heat treatment. The phase kinetics on the nanosecond to microsecond scale were studied using the Dynamic Transmission Electron Microscope (DTEM), an instrument uniquely capable of capturing nine transmission electron images or diffraction patterns from a sub-micrometer piece of material in one microsecond $[3,4]$. Pulsed-laser heat treatment in the DTEM produced a relatively subtle changed in the [100] zone-axis diffraction pattern, whereby spots with odd values of $k+l$ (which are forbidden in pure $\mathrm{MoO}_{3}$ ) get dimmer (Fig. 1 (a-b), red circles), while spots with both $k$ and $l$ even get stronger (green squares). Selected frames from a 9-frame time-resolved diffraction experiment (Fig. $1(\mathrm{c})$ ) show that this change begins after a roughly $500 \mathrm{~ns}$ incubation time and is largely complete within the next 500 ns. Provided the laser intensity is below the threshold for damaging the material, further pulses simply strengthen this pattern and cause no further transformations. Real-space reconstruction from static diffraction patterns (Fig. 2) of the laser annealed structure produced a model in which the symmetry of the unit cell is reduced so as to make way for an ordered layer of copper atoms.

More conventional in situ TEM heating experiments, on a time scale of minutes rather than microseconds, never produced this structure. Instead, they produced a completely different annealed structure characterized by superlattice spots (Fig. 1(d), red circles) and satellite peaks at incommensurate positions (blue arrows), consistent with the formation of an incommensurate charge 
density wave with a period of several unit cells. This is striking, because the formation of charge density waves in 2D layered materials is normally a picosecond-scale phenomenon; the presence of copper seems to have slowed it down by many orders of magnitude. Control experiments with pure $\mathrm{M}_{0} \mathrm{O}_{3}$ showed none of these transformations, demonstrating that the copper is playing an essential role in the phase evolution.
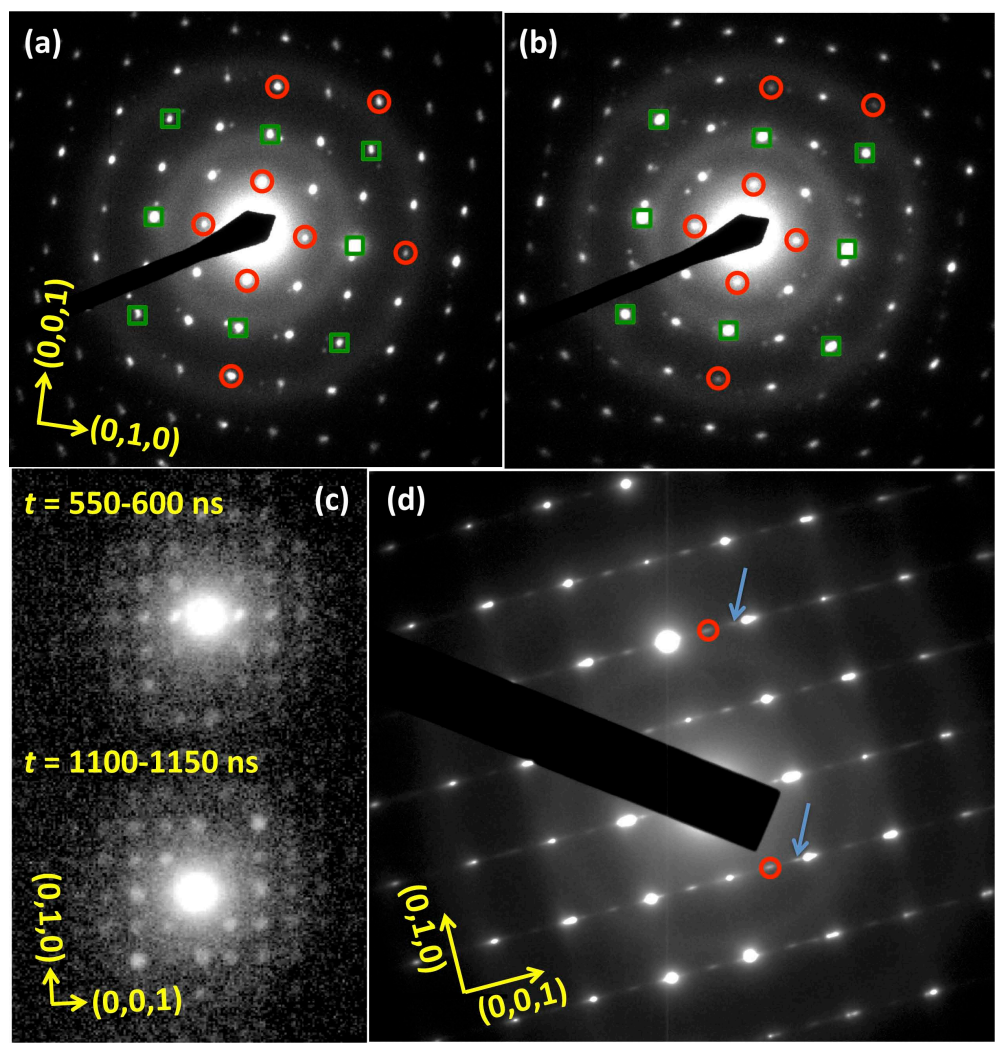

Measured Diffraction Pattern

Figure 2. An example reconstruction result, matching the discretized $(0, k, l)$ spot intensities in a [100] zone axis pattern to high precision in order to determine the structural rearrangement in Fig. 1(c).
Figure 1. Diffraction patterns of transformations in $\mathrm{Cu}$-intercalated $\mathrm{MoO}_{3}$ nanoribbons. (a)-(c) are Dynamic TEM experiments with 12 ns heating times and $\sim 10 \mu$ s characteristic cooling times, (a) Before heat treatment, (b) After heat treatment, showing a change in relative spot intensities indicative of a structural rearrangement within the unit cell, (c) Selected diffraction patterns from a time-resolved experiment using 50 ns exposure times, (d) A completely different structure resulting from heating on a time scale of several minutes, with incommensurate satellite spots indicative of ordering on the scale of multiple unit cells.

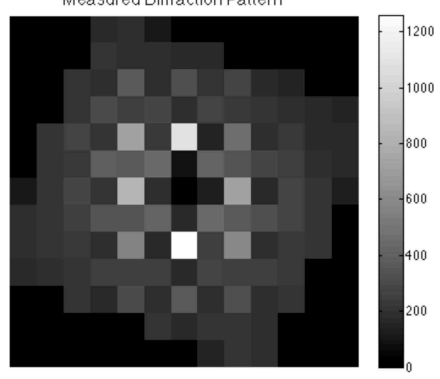

Simulated Diffraction Pattern

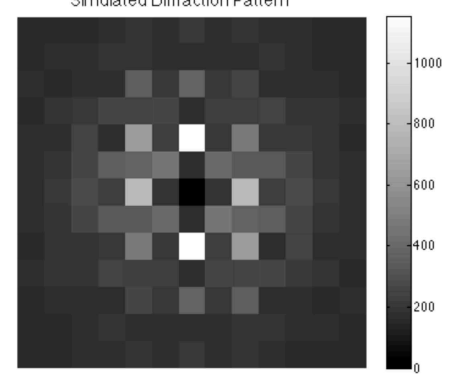

[1] K. J. Koski et al, J. Amer. Chem. Soc. 134 (2012), p. 7584.

[2] K. J. Koski et al, J. Amer. Chem. Soc. 134 (2012), p. 13773.

[3] T. LaGrange et al, Ultramicroscopy 108 (2008), p. 1441.

[4] M. K. Santals et al, App. Phys. Lett. 102 (2013), 174105.

[5] This work performed under the auspices of the U.S. Department of Energy, Office of Basic Energy Sciences, Division of Materials Sciences and Engineering by Lawrence Livermore National Laboratory under Contract DE-AC52-07NA27344. 